\title{
Peculiarities of translation of uncodified vocabulary
}

\author{
Elena Gaylomazova ${ }^{1,3}$, Oksana Dyshekova ${ }^{2,3}$, Vladimir Sarkisyants ${ }^{4}$, and Anna \\ Kudryashova $^{5,2}$ \\ ${ }^{1}$ K.G. Razumovsky Moscow State University of Technologies and Management, 109004, Moscow, \\ Russia \\ ${ }^{2}$ Russian Customs Academy, Rostov branch, 344002, Rostov-on-Don, Russia \\ ${ }^{3}$ Don State Technical University, 344000, Rostov-on-Don, Russia. \\ ${ }^{4}$ Russian State University of Justice, Rostov branch, 344038, Rostov-on-Don, Russia \\ ${ }^{5}$ Southern Federal University, 344006, Rostov-on-Don, Russia
}

\begin{abstract}
Nowadays, linguists invariably show a great interest in the whole variety of uncodified vocabulary. Researchers view the language as a kind of "mirror" of the processes and transformations taking place in society, since it is in the language that reflects the current state and development of the human community. It is the language and all its lexical composition that most vividly reacts to what is happening in society through transformations and changes in structure and functioning. Nonstandard words and expressions are now increasingly serving as means of expressing the positive emotional mood of the author of the statement, and their use in speech contributes to the optimization of communicative interaction in the context of informal interpersonal communication. In modern linguistic literature, as in society as a whole, one can observe an ambiguous attitude towards the problem of active penetration of words and expressions of uncodified vocabulary into various styles of speech, there is no unified approach to assessing the ongoing transformations in determining the place and the function of uncodified vocabulary in the linguistic and communicative space. An attempt is made in the work to identify the features of the functioning and translation of uncodified vocabulary from English into Russian.
\end{abstract}

\section{Introduction}

The modern stage of development of human society is characterized by the rapidly proceeding democratization of all spheres of human life, which ultimately leads to an increase in freedom of speech and self-expression. The results of the active promotion of this freedom are also reflected in the linguistic aspect: words and expressions that previously belonged to the layer of indecent and even obscene, can increasingly be heard in the speech of representatives of different segments of the population, from television

*Corresponding author: elena.gailomazova@yandex.ru 
screens, on the radio, in public places, in public speeches of politicians and other public figures, representatives of mass culture, in the media. According to the researchers, this phenomenon can be explained by the acquisition of a new functional load by profanity, which was unusual for her several decades ago. Non-standard words and expressions are now increasingly serving as a means of expressing the positive emotional mood of the author of the statement, and their use in speech contributes to the optimization of communicative interaction in the context of informal interpersonal communication. Researchers associate the active expansion of the scope of use and functioning of substandard vocabulary with the process of rethinking established communicative and spiritual and moral values taking place in modern society, which entails a significant impact on the existing linguistic norm.

In modern linguistic literature, as in society as a whole, there is an ambiguous attitude towards the problem of active penetration of words and expressions of uncodified vocabulary into various styles of speech, there is no unified approach to assessing the ongoing transformations in determining the place and function of uncodified vocabulary in the linguistic and communicative space. Taking into account the constantly increasing process of globalization, it seems relevant to comprehensively consider slang in a comparative aspect on the material of several languages, for example, English and Russian. Research in this direction, in our opinion, will significantly improve the linguistic competence of native speakers of the Russian language, including those who study English, will make a certain contribution to the development and improvement of the culture of speech, as well as the formation of communicative tact, a sense of the communicative expediency of using slang units in various conditions of interpersonal and intercultural communication.

\section{Materials and methods}

According to the currently established concepts of modern linguistic science, the linguistic system is a set of linguistic elements that are in certain relationships with each other and enter into various connections with each other. The result of all the diversity of relationships and connections is the formation of a certain unity and integrity. Each linguistic unit functions in the system as a part of the whole enters into a certain connection with other units and parts of the linguistic system, directly or indirectly, that is, through the categories that exist in the language. A language system is a complex object and has a multidimensional structure both in use and in the development process. Throughout the history of the existence and study of the language, vocabulary and its research have invariably attracted increased attention of scientists.

\section{Results}

Language is inseparably linked with the social development of the individual and society. The language instantly reflects the state and development of society at a definite stage of its development. Language is very sensitive to transformations taking place in society, the linguistic markers of which are changes in the structure, functioning and composition of the vocabulary. The explanation for this phenomenon, as the researchers note, should be sought in new functions implemented in substandard vocabulary.

When classifying the vocabulary of the language, many researchers distinguish such groups of vocabulary as: 1 . neutral vocabulary, 2 . literary and book vocabulary, represented by such types as poetisms, archaisms, historicisms, terms, etc., 3. non-literary vocabulary 
that covers non-literary colloquial words and expressions, dialectisms, colloquial vocabulary, jargon, professionalism, vulgarism.

Uncodified vocabulary, which includes a whole complex of non-literary vocabulary, is an integral component of the lexical system of the language.

Non-literary or reduced vocabulary can be divided into two main subgroups according to the parameter of the degree of inherent expressive and stylistic coloring: colloquial and vernacular vocabulary.

The main sphere of use of colloquial vocabulary is casual communication carried out in an informal setting. Words of this group can contain an assessment of the designated object, property or action. According to the parameter of part-of-speech affiliation, the volume of colloquial vocabulary traditionally includes words of significant and service parts of speech: nouns (non-sense, flick, celeb, moniker, teen, ciggie); adjectives (weird, nutty, bullheaded); verbs (to wow, to OK, to boost); adverbs (to psy-turvy, a bit, hobandnob); interjections (oh, ouch), etc.

The main difference between vernacular lexicon and lexical units of colloquial lexicon is a higher degree of actualized expressive coloring. Units of vernacular vocabulary are used, as a rule, in conditions of emotionally intense situations of interpersonal communication. The use of vernacular words and expressions serves the purpose of expressing the evaluative or emotional attitude of the author of the statement to the nominated person, to give an evaluative characteristic of the property, phenomenon. This vocabulary is typical for friendly and familiar relations between participants in communication. The vernacular group of vocabulary includes such words and expressions as wally, simple (about a person), chump; clown, etc.

A special group of vernacular vocabulary is made up of rude and vulgar words and expressions: psycho, idiot, sucker. The use of this vocabulary can be carried out in conditions of conflicting communication, when the speaker aims to humiliate, offend the interlocutor.

Considering the lexical system of the English language as a socio-communicative system, the researchers propose to divide the entire vocabulary of the language into three complex lexical tiers, which oppose each other in such a parameter as the correspondence of the lexical unit, exists at a certain stage in the development of the linguistic community to the sociolinguistic norm. The following lexical tiers are traditionally distinguished:

- literary standard;

- expressive vernacular;

- social and professional vernacular.

Each of the selected lexical tiers is further subdivided into three lexical levels, which form the basis of the speech repertoire of the corresponding register in the implementation of communication.

Each of the allocated lexical tiers is further subdivided into three lexical levels, which form the basis of the vocabulary of the corresponding register in the implementation of communication (Fig. 1) 


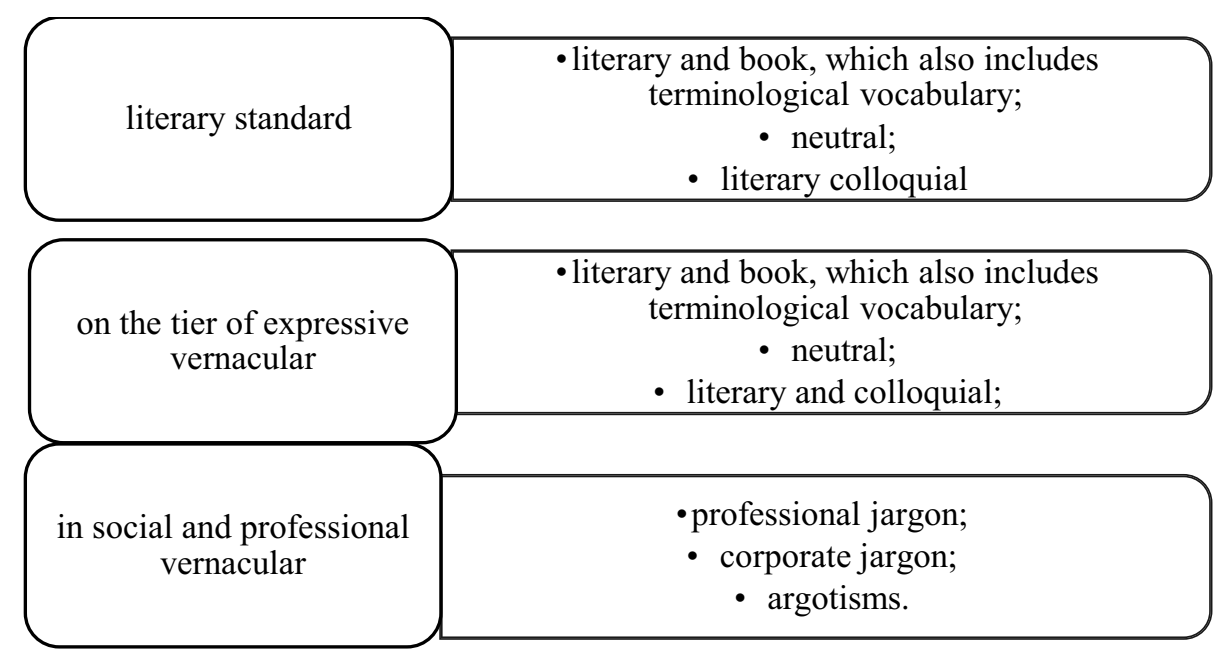

Fig. 1. Levels of lexical tiers

A distinctive feature of low colloquial forms, general slangisms and vulgarisms, that is, units of expressive vernacular, is the absence of social and professional marking, which is considered by researchers as their main difference from the units of professional vernacular.

Vernacular is an obligatory component of any natural language. Vernacular units are used in oral communication, from which they can be borrowed into literary language. Vernacular, being the basic form of the national language, has its own distinctive characteristics. This is, on the one hand, the lack of proper features of the systemic organization, and on the other hand, the presence of a special stock of linguistic forms that violate the norms of the literary language.Violation of the norms of the literary language, according to the authors, as a rule, is not recognized by those who use vernacular units in their speech.

The concept of "national language" includes, at the same time a standardized literary language and territorial and social dialects that are outside the literary norm. Social dialects mean professionalisms, jargon and slangisms, as well as vernacular. The classification of social variants of speech, proposed by V.D. Bondaletov, is as follows:

1) professional languages, for example: fishermen, hunters, potters, woodworkers, wool, shoemakers, as well as representatives of other trades and occupations;

2) group, or corporate, jargons, for example, the jargons of pupils, students, athletes, soldiers and other, mainly youth, collectives; conventionally professional languages of artisans and social groups close to them;

3) conventional declassed languages [2].

In accordance with the proposed classification, conditional languages and jargons of declassed elements belong to different types of social dialects. The main difference lies in the sphere of the function they perform: the former perform mainly a conspiratorial function, the latter - emotionally expressive.

V.V. Himikalso distinguishes between the concepts of argot and jargon. "Argo is a closed lexical system of special nominations serving narrow social and group interests, primarily professional ones. Argotisms - rational nominations - terminoids (similar to terms) used in the practical interests of the profession, craft, business" [8].

Jargon, in comparison with argot, is a more open subsystem, while slang is an almost open subsystem of non-normative lexical and phraseological units of the colloquial language. 
Jargonism is easily recognizable and more or less clear to everyone, for this it is used. Using a jargon word, the speaker manifests or imitates his belonging to a particular social group and expresses his attitude to the environment - to objects or partners in speech - from the position of this social group. "Unlike argot and jargon, slang does not have a distinct social and group orientation: it can be used by representatives of different professions, different social and educational status, and even different ages" [1].

At present there is no unified approach among researchers regarding the functions inherent in uncodified vocabulary.

In general, the following are recognized as the main functions of uncodified vocabulary identified by most researchers of this layer of vocabulary: communicative, cognitive, nominative, expressive, ideological, esoteric (conspiratorial), identification, as well as the function of saving time (linguistic economy) [4].

Currently, almost every new film uses a lot of obscene expressions. We refer profanity to the colloquial category and is a segment of abusive vocabulary of various languages. The use of uncodified vocabulary by a person is due to different reasons. The use of uncodified vocabulary by a person is due to various reasons, such as a teenage desire to stand out, or an emotional state at a certain moment (anger, rejection). For certain people, obscene language is an integral part of their vocabulary.

The translation of uncodified vocabulary based on its inherent bright stylistic and expressive-emotional coloration, as well as national color, makes this lexical layer rather difficult to translate into one or another language. Individual elements may even refer to non-equivalent vocabulary and be considered "untranslatable".

Although the translation is intended to fully replace the original, its complete identity with the original text is unachievable and we can only talk about equivalence, that is, some degree of closeness of the translation to the original.

Obscene vocabulary is distinguished by clear national-linguistic and social specifics, which are especially clearly manifested when comparing the used units of obscene vocabulary in different cultures. In our work, we make an attempt to highlight the specifics and possible difficulties of translating the above-mentioned vocabulary from Russian into English when translating the feature films.

When considering the issue of uncodified vocabulary, the question immediately arises of the motivation of the reasons for the speakers to use stylistically colored units in speech. It is impossible to deny the fact that the use of profanity is the deliberate use of elements of the general literary dictionary in colloquial speech for purely stylistic purposes. The main task is to create the effect of difference from recognized samples, giving liveliness and expressiveness to the statement.

And, of course, one cannot fail to note one equally important reason for the use of uncodified vocabulary - this is the demonstration of a certain linguistic competence. In modern society, obscene vocabulary used in certain situations is not, often, something unacceptable. The competent use of units of speech related to this register is an indicator of the cultural level, and not the absence of culture.

\section{Discussion}

All the above-mentioned features of non-literary vocabulary allow us to conclude that today its units are difficult when considering them from a linguistic point of view. This fact must be taken into account when working on the translation of slang vocabulary from one language to another.

The main problem in translating obscene vocabulary is the discrepancy in the composition of lexical-semantic groups that provide slang and vernacular for Russian and English. 
Another reason that causes difficulties in translating obscene vocabulary is the deviation in the pragmatic and semantic components of the meaning of words that are equivalent in their lexical and semantic variants. This leads to a discrepancy in the results of the lexical and semantic variation of these words in Russian and English.

Another no less important reason for difficulties in translating uncodified vocabulary from Russian into English is the components of uncodified vocabulary: vernacular, argot, jargon, slang. subject to frequent changes. The translator also needs to keep in mind the time factor. Many slang words are the speech mark of a certain generation, and inappropriate words threaten to turn into anachronisms.

To achieve an adequate translation, it is necessary to know the ethno cultural characteristics of the people speaking the language from which the translation is carried out.

The main problems of the theory of translation at the present stage of the development of linguistic science is the problem of transferring the communicative effect of the original language. Within the framework of this problem, the issue of translation of stylistically colored vocabulary units is of particular interest.

Each translator strives to convey the stylistic features of the source language as accurately as possible. The starting point for translating profanity in the original language is to search for all sorts of equivalents of profanity units in the translating language.

There are several main ways to translate obscene Russian vocabulary into English. First, the two main paths followed by the translator are direct and functional (indirect) translation. According to E.V. Breus, direct translation includes techniques such as transcription, transliteration, tracing, and semantic neologism. Functional translation includes equivalent, functional analog and descriptive translation. As for the direct method, it is rarely used when translating units of uncodified vocabulary, since this violates the principles of translation adequacy and the usual norms of the target language. However, there are some cases where the choice of this translation method is the only way out. Each individual case depends, of course, on the context [3].

Translators also resort to indirect methods of translation or translation transformations. Their main function is to create a lexically adequate translation of a work in the absence of linguistic correspondence. It must be noted that an adequate translation is impossible without taking into account the stylistic side of the original, since the translation creates a stylistic analogue of the original text.

Lexical substitution is a transformation consisting in the substitution of individual lexical units of the original language with the lexical units of the translator, which are not their dictionary equivalents, which have a denotative meaning, in contrast to the units of the original language transmitted by them in translation. The following transformations are most common: generalization, euphemistic translation, compensation, omission, etc.

The following lexical transformations are the most frequent.

Generalization.Generalization is the replacement of a unit of the source language, with a narrower meaning, with a unit of the translating language with a wider meaning.

English vocabulary is more abstract than Russian words denoting the same concept. Specialists use less frequently when translating from English into Russian than when translating back. When translating uncodified vocabulary from Russian into English, generalization is one of the main types of translation:

Compensation. This method is used in cases where certain text elements in the source language do not have an equivalent in the target language and cannot be transmitted using it. In these cases, the translator conveys the same information in a different way and not necessarily in the same place as in the original text in order to compensate for the semantic loss caused by the fact that one or another unit of the original language was not translated or the translation was not completed in full. 
Omission. The technique in which lexically and semantically redundant words are removed from the text is called omission. These words can be such grammatically redundant elements as articles, possessive pronouns or lexemes, especially paired synonyms.

Replacing the effect with the cause and vice versa. In the process of translation, lexical substitutions based on causal relationships between concepts often take place. Transformation, in which a word or phrase of the original language can be replaced during translation by a word or phrase of the translating language, which logically denotes the cause or effect of an action or state indicated by the unit of the source language being translated.

Euphemistic and Dysphemistic Translation. These techniques are used when obscene language, vulgarisms and jargon are encountered in the original text.

Euphemistic translation consists in replacing the words of the original with a strong or crude expression with words with less strong expression in the translation.

The dysphemistic translation, which is the opposite of the euphemistic one, is the replacement of a word from the original text with a less vivid expression when translated into a rougher one.

Thus, we can conclude that translation techniques, including transformations, are decisions taken in the context of the context. The main task is to achieve the maximum level of adequacy and accuracy of the transfer of meaning, in the text of the translation in the absence of exact matches in the target language that convey the content and stylistic side of the work.

The comparative analysis of the translation of uncodified vocabulary from Russian into English undertaken within the framework of this study indicates that errors and inaccuracies are inevitable when translating any material, both oral and written, since it is impossible to translate an authentic text with one hundred percent accuracy. In any case, the translator will be confronted with the untranslatable, and the quality of the final version will depend on the translation decisions he makes. Unfortunately, these solutions are often not entirely successful.

As material for the practical part of our work, we took excerpts from several modern Russian films that are closest to modern realities. As you know, the speech of movie characters is as close as possible to lively spoken language, so we believe that the study of techniques for translating profanity will help to expand the scope of knowledge in this area of linguistics.

When analyzing the techniques for translating obscene vocabulary, we will compare the replicas of the characters in the original language with an existing duplicate translation or with our own.

Table 1. Prison jargon

English variant
- At the meeting of bandits we have come to the agreement.
Russian variant
- Na razborke dobazarilis' po-mirnomu.

Here we see the verb "dobazarilis"', which refers to prison jargon and has the meaning "to agree." In the above translation, it can be seen that we used a descriptive translation of this meaning and received the expression "to come to the agreement".

In the same example, there is one more unit of uncodified vocabulary. A synonym for the word "razborka", which is used as criminal jargon, will be the expression "to sort things out." In this example, we use a descriptive translation like "the meeting of bandits", neutralizing the expressiveness of Russian jargon. 
Table 2. Argotizm

English variant
- Stay on the market all day long and don't catch his eye.
Russian variant
- Den' i noch' torchite na rynke, na glaza emu ne popadajtes'.

In this example, we are considering the verb "torchite", which is and refers to thieves' jargon, meaning "to be somewhere for a long time." In our translation, we neutralize the figurativeness of expression, using generalization, we use the verb "to stay". Consider the following example:

Table 3. Vernacular

English variant
- Sup boozers, how's the trade? Russianvariant
Russian variant
- Nu, chto, sinyaki, torguem?

Here we see an expression referring to obscene vocabulary, "sinyaki", applied in relation to persons who are addicted to alcohol. When translating, we use the full equivalent of this unit in the translating language "boozers".

Table 4. Slang

\begin{tabular}{l} 
English variant \\
- Someone has ratted some money. Gotta get it back. \\
- So can I also take part? \\
- Have a gun? Or are you going to use that? \\
Russian variant \\
- Da chelovechek odin deneg skrysil. Nado vernut'! \\
- Tak, mozhet, iya $v$ dele pouchastvuyu? \\
- Volyna imeetsya ili ty vot s etim sobiraesh'sya? \\
\hline
\end{tabular}

Consider such a lexical unit as the slang verb "skrysil" - it means "to steal". When translating this verb, you can use the generalization method and interpret it as "to steal", but in colloquial English there is a verb "to rat", the meaning of which is clear based on the meaning of the noun "rat", respectively, the verb will be the full equivalent of the Russian lexeme.

The second stylistically diminished unit in this example is "volyna", which is jargon and means "weapon". In our case, we neutralized the word given to us and used in translation such an equivalent as "a gun".

Let's move on to the next example

Table 5.Thieves' jargon

\begin{tabular}{l|}
\hline English variant \\
- Mr. Romashkin is asking for the spoon. \\
- What oar? \\
- Have you any oar? \\
Russian variant \\
- A vesla net? \\
- Kakogo vesla? \\
- Viktor Sergeevich lozhku prosit. \\
\hline
\end{tabular}


In the above case, we consider the jargon "veslo"(paddle) which is interpreted from thieves' jargon as a cutlery, or rather a "spoon". Due to the lack of a lexeme equivalent in English, based on the context, we can conclude that in this example there is no need to look for an equivalent or an analogue, since what follows is an explanation of the meaning of jargon. Following this, we used the word "an oar", which means a wooden rowing tool

When dubbing a film, the method of selection of a functional analogue and equivalent, generalization and neutralization of uncodified expressions, the method of omitting part of the information is used, which is due to the temporal limitation of the sound. The corpus of factual material also presents such translation methods as compensation and replacement of a cause by an effect and vice versa.

After analyzing translation transformations, we came to the conclusion that the preferred translation methods were: euphemistic translation - $20 \%$, compensation - $40 \%$, specification $-32 \%$, generalization $-21 \%$, method of equivalent lexical replacement $-25 \%$. Specialists in the field of translation have also turned to dysphemistic and antonymic translation $-10 \%$, the method of omission $-12 \%$, addition- $11 \%$, tracing- $7 \%$ and, in the rare case, to descriptive translation $-10 \%$. Here is a diagram that clearly shows the frequency of the use of different ways of translating slang in cinematography.

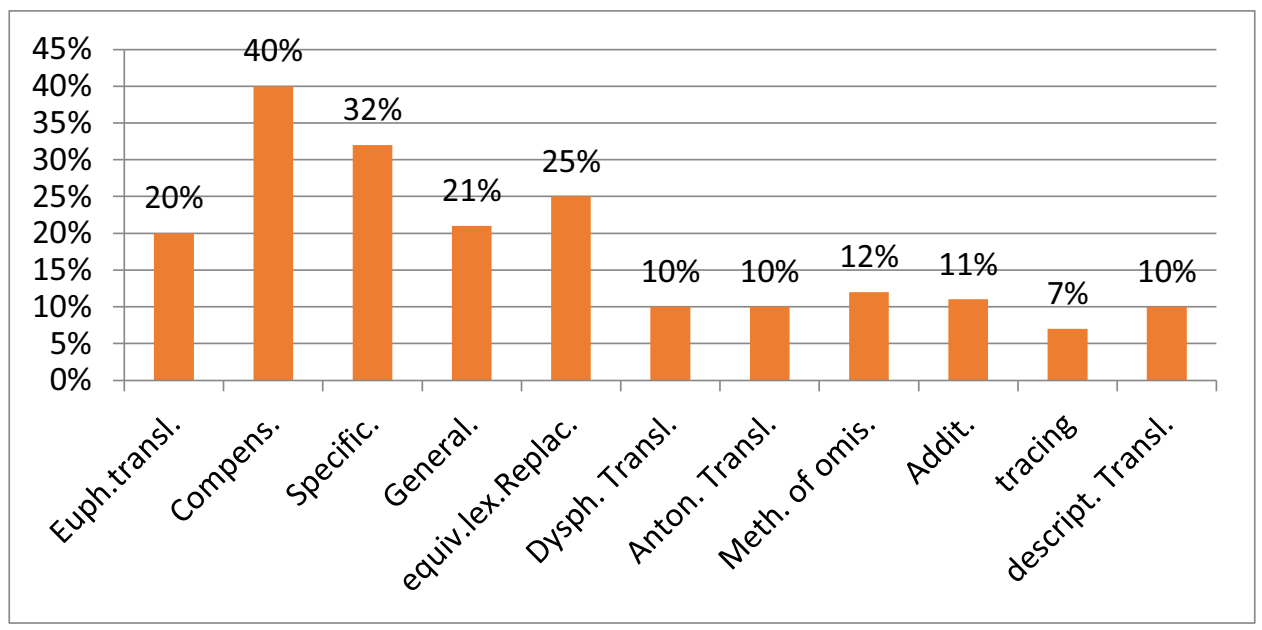

Fig. 2. Analyzing of translation transformations

This diagram clearly shows that the most common techniques for translating slang in cinema are the compensation method, the concretization method and the generalization method. The frequency of using these translation transformations is explained by the fact that slang is an integral part of the reduced vocabulary of young people, which is characterized by high expressiveness, pragmatism, and semantic load. When translating youth slang and youth subcultures, the translator must take into account many factors that may motivate the use of a particular expression. A comparative analysis of the translation showed that the translation of slang units into Russian is based on a situational factor, and not on the register of a certain genre. The slang translation boundaries shown in this series have imprecise linguistic boundaries, suggesting translation techniques such as transforming lexemes from one lexical register to another and sometimes full translations showing a variety of syntax and sentences using different translation methods of youth slang and culture.

It would be unfair to reduce the discussion of uncodifiedvocabulary to argotisms, vulgarisms and colloquial speech. Professional spoken language exists among representatives of various specialties, including the environmental sphere. 
Among all the variety of uncodified vocabulary, professional jargon prevails. Frequent use of words in a certain environment contributes to the violation of linguistic rules and norms and is fertile ground for the appearance of jargon. Among the main ways of formation of professionalism are: 1. Abbreviation: $\mathrm{AQS}=$ air quality standard; 2 . Combining two or more words or their parts into one: pipe + line $=$ pipeline, after + regeneration $=$ after regeneration ("forest restoration"). 3. The use of words that are not directly related to the professional field of activity as metaphors. The word "casual ecologist"is used to refer to freelancers for an environmental company.

\section{Conclusions}

Analysis of argot, jargon and slang has shown that their status and boundaries in the English language system remain undefined in modern linguistics. The content of these concepts is interpreted by different scientists almost synonymously, which is clearly reflected in the classifications presented by them. In this study, we accept the opinion of those researchers who distinguish between the concepts of jargon, argot and slang.

Currently, there is no general approach to defining the essence of youth slang. A number of scientists completely deny the existence of slang as an independent subsystem in the system of the national language, referring it to jargon, then to argot, then to dialectism. In this work, we are of the opinion that slang is an independent subsystem of the national Russian and English languages, which implements both common for the entire language and functions characteristic exclusively for it, as well as having its own distinctive features in linguistic, stylistic and pragmatic plans. Slang is understood as a type of speech used mainly in oral communication of a separate relatively stable social group that unites people on the basis of profession, position in society, interests or age.

The analysis ofthe slang vocabulary carried out in the work, showed that the main difficulty in translating slang units is understanding the meaning of a new word. In the final version, the translation should convey both the semantic content and the expressive-stylistic coloring of the word.

As a result of our research, we came to the conclusion that when translating slang units, one should focus primarily not on bilingual dictionaries, but on explanatory and specialized dictionaries of the receiving language, since they contain more accurate information. When working with slang vocabulary, the translator must have sufficient knowledge of the substandard to which the slang belongs, have background knowledge and be able to work with various kinds of reference literature to achieve the adequacy of the translation.

\section{References}

1. Irina Alekseeva, Introduction to Translation Studies, (2014).

2. Vasily Bondaletov, Social Linguistics, (2012).

3. Eugene Breus, Theory and practice of translation from English to Russian, (2017).

4. Coleman, J. The Life of Slang, (2012).

5. Cristopher D. Broyles, Toxicology and Applied Pharmacology 254(2), 215-220 (2011). doi.org/10.1016/j.taap.2010.10.015

6. Eneko Antón, Jon Andoni Duñabeitia, Behavior Sciences 10(8), 123 (2020). doi.org/10.3390/bs100801234

7. Eric M. Anicich, Adam D. Galinsky, Organizational Behavior and Human Decision Processes, 161, 274-290 (2020). doi.org/10.1016/j.obhdp.2020.07.001

8. Vasily Himik, Poetics of Low, or Urban Vernacular as a Cultural Phenomenon, (2001) 
9. 9. Sergey Kuznetsov, Big Explanatory Dictionary of the Russian Language, (2014).

10. Vladimir Matyushenko, Dictionary of English Slang, (2002).

11. Lev Nelyubin, Explanatory Translation Dictionary, (2008).

12. Rosa Giménez-Moreno, Hanna Skorczynska, Procedia - Social and Behavioral Sciences 95, 402-408 (2013). doi.org/10.1016/j.sbspro.2013.10.662

13. Soudabeh Nour, Esli Struys, Hélène Stengers. Behavior Sciences 9(4), 43 (2019). doi.org/10.3390/bs9040043

14. O'Grady, W.,Contemporary Linguistics, (2001).

15. Urban Dictionary, http://www.urbandictionary.com. 